\title{
LA MULTIPLICACIÓN DE LOS PANES EN LA EUCOLOGÍA HISPANA. ESTUDIO DE UN FORMULARIO OLVIDADO
}

\author{
POR \\ ADOLFO IVORRA ROBLA \\ Facultad de Teología San Dámaso, Madrid
}

\section{RESUMEN}

La eucología no discurre siempre por los mismos estilos ni por las mismas temáticas. Con la unificación de los libros litúrgicos y la extensión del rito romano, con su conocida concinitas eucológica, las oraciones litúrgicas aparecen con esquemas y temáticas parecidas. La prodigalidad de los textos hispanos o la tipología es dejada de lado. Sin embargo, la eucología hispana, incluso en sus características propias (Spanish symptoms), se nos muestra siempre original. En el formulario aquí estudiado, se nos presenta la oración litúrgica en continuidad con la exégesis espiritual que encontramos en los Padres. La multiplicación de los panes es un tema oracional pero también es un recurso para mostrar la unidad entre los dos Testamentos, además de concebir la oración en continuidad con la reflexión teológica.

PALABRAS CLAVE: Eucología, Liturgia hispana, Exégesis litúrgica, Exégesis bíblica, Milagros.

\section{ABSTRACT}

The eucology doesn't follow the same styles or the same themes. With the unification of the liturgical books and the expansion of the roman rite, with its well known eucological concinitas, liturgical prayers appears with common schemes and thematics. The abundance of the hispanic texts or the typology are putted a side. However, the hispanic eucology, including its proper characteristics (Spanish symptoms), is always original. In the studied formulary here, the liturgical prayer presents us in continuity with the spiritual exegesis that we found in the Fathers. The multiplication of breads is a praying theme but also a recourse for to show the unity between the two Testaments, besides to conceive the pray in continuity with theological reflection. 
KEY WORDS: Eucology, Hispanic liturgy, Liturgical exegesis, Biblical exegesis, Miracles.

Recibido/Received 29-12-2006

Aceptado/Accepted 04-02-2007

Con la renovación del misal hispano se intentó ofrecer lo genuino de la tradición hispana, a la vez que se procedió a rescatar formularios que quedaron olvidados con el tiempo. Sin embargo, en algunos casos muy precisos, la renovación del misal implicó una selección de textos que implicaba la desaparición de algunos, ya sea por su carácter tardío, por su estructura literaria o por su temática específica. En el caso del Missale Hispano-Mozarabicum (=MHM), esto significó una serie de cambios en un tiempo litúrgico que equivale al per annum romano, el tiempo De Cotidiano. Este tiempo litúrgico, desaparecido en el segundo milenio por la adaptación del calendario hispano al romano, reaparece en el actual misal, fruto de los trabajos de Jaime Sancho Andreu, que estudió los formularios de este tiempo presentando en $1981^{1}$ una serie completa de 26 misas. En el actual MHM, en su primer volumen (1991), encontramos 17 misas $^{2}$. Por tanto, podemos decir que del ciclo de Tempore, el tiempo De Cotidiano fue el que «sufrió» más cambios o, desde otro punto de vista, (re-)nació de forma limitada y cíclica ${ }^{3}$. No es momento de entrar en las cuestiones que llevaron a concebir el tiempo De Cotidiano tal como lo encontramos hoy, sino de volver a presentar esos textos que después de la renovación no encontramos en el actual misal, cuya temática y forma pueden ser interesantes para los estudios sobre eucología 4 .

Los textos que estudiamos corresponden en la edición crítica de J. Sancho a la misa Discussa paululum, formulario 20. Su origen está en el manuscrito S6 de Silos, que presenta cierto desorden y errores de transcripción. No lo encontramos en el Missale Mixtum, por lo que el formulario en cuestión no ha sido utilizado en mucho tiempo.

Tal como afirma J. Sancho, la Oratio Admonitionis -que en el manuscrito original aparece como Alia-, la Ad Pacem, la Illatio y el Post Sanctus comparten la misma temática, el milagro de la multiplicación de los panes y los peces ${ }^{5}$. J. San-

\footnotetext{
${ }^{1}$ Los formularios eucarísticos de los domingos de quotidiano en el rito hispánico. Edición crítica y estudio teológico, Valencia, 1981. Anteriormente ya se había introducido en el tema: Cfr. «Los formularios eucarísticos de los domingos de quotidiano en el rito hispánico», en AA. VV., Liturgia y música mozárabes, Toledo, 1975, 87-104.

${ }^{2}$ Los Prenotantos (n. 153) se equivocan al afirmar que existen 18 series de textos eucológicos.

${ }^{3}$ Desde el domingo diecisiete el MHM incluye una rúbrica que tiene el esquema siguiente: Pro oratione admonitionis et aliis, quaere superius in ... dominico, $p . .$.

${ }^{4} \mathrm{La}$ ausencia de esta illatio implica la ausencia de términos latinos en el misal, que sólo encontramos aquí.

${ }^{5}$ En el actual misal el tema de la multiplicación aparece en la illatio de la misa In Apparitione Domini.
} 
cho también señala la más que probable hipótesis de que el formulario se habría compuesto a partir del evangelio del día ${ }^{6}$. Además de la forma poco usual de la illatio, la causa de que este formulario no fuera reeditado para la actual edición del misal hispano se deja entrever en el contenido de los textos del formulario, donde en mayor o menor medida, la 'alegoría' se hace presente. Esto no sorprende, teniendo en cuenta la amplia influencia que tuvo Jungmann al descalificar la alegoría -que no siempre se distingue bien de la tipología- en la liturgia, donde no se escuchó la voz de Henri de Lubac que estudió la alegoría dentro de su ámbito propio: la exégesis medieval de la Sagrada Escritura7. En su estudio, Lubac recoge el sentido propio de la alegoría junto a los otros tres sentidos de la Escritura, según la expresión más extendida: Litera gesta docet, quid credas allegoria, Moralis quid agas, quo tendas anagogia ${ }^{8}$. Desde este punto de vista, la alegoría no se refiere a algo fantasioso ni ajeno a la fe. La Post Nomina de nuestra misa nos advierte ya sobre el sentido del método que se va a usar:

\section{Primum orationem difussam}

secreta cordis obscuritas excutientes irae

ut spiritales illius divinae intelligentiae interpretationes,

lectio premissa impellit,

eodem Domino discurrente,

nuntiis vestris favoris adiutos,

et induti sensos explicationem disponamus;

ut et populi sensum intelligentiae capiant,

et holocaustum nostrum pius Dominus

in odorem suavitatis accipiat,

et defunctis fidelibus

requies praestetur aeterna

J. Sancho afirma que esta oración se ajusta al género propio de las Post Nomina al final, y que la primera parte «parece el fragmento inicial de una Oratio admonitionis, con la explicación de cómo se debe pasar bien dispuestos por la liturgia de la Palabra a la oración y al sacrificio» ${ }^{9}$. Esto adquiere mayor fuerza si pensamos en cómo califica la Oratio Admonitionis de la misa a la perícopa

\footnotetext{
${ }^{6}$ Cfr. J. SANCHO, Los formularios eucarísticos de los domingos de quotidiano en el rito hispánico, 99.

${ }^{7}$ Cfr. H. DE LuBAC, Exégèse médiévale. Les quatre sens de l'Écriture, I-IV, Paris, 1959-1964.

${ }^{8}$ Cfr. H. DE LuBAC, Exégèse médiévale, I, Paris, 1959, 23.

9 J. SANCHO, o. $c ., 100$.
} 
que llama nuestra atención: mystica evangeliorum pagina. Además de esto, en la Post Nomina se ve la comprensión que el autor tiene de la Sagrada Escritura y de su interpretación, de la cual es imposible no establecer paralelismos con la intencionalidad del método de los cuatro sentidos de la Escritura. En efecto, se alude a la comprensión de las spiritales interpretationes, al sensum intelligentiae, en unión con el ofrecimiento del piadoso holocausto a Dios. Acción litúrgica y ruminatio de la Escritura se conciben, de este modo, como dos realidades incluyentes ${ }^{10}$.

Sin embargo, la comprensión espiritual del posible evangelio del día (Mc 6, 38ss) se realiza de forma gradual. En la mencionada Oratio Admonitionis apenas se hace referencia al milagro ${ }^{11}$. Del mismo modo que en los comentaristas medievales en sus expositio missae, la Ad Pacem intenta asociar ambos testamentos por medio de la tipología ${ }^{12}$ :

Mirabilia tua, Domine, trementes narramus,

duo sub inde pisciculi

veteris ac novi indicant testamentorum mysteria:

primum quod Tobi Tobiae filius iubente Rafaelum

vadoso amni decurso continuit,

cuius caro pastum sumministravit,

itineris successu iecur vetustum

a Filia Raquelis demonium effugavit,

a ruina quoque eius Tobiae occulos

caliginis nubes contactos

sereno lucis aspectu

ad tactum unctionis infuit,

hoc est:

ut ignorantiam primi saeculi

${ }^{10}$ De este modo, la lex orandi «ofrece la palabra santa de Dios a su modo; caldeada al amor de la meditación multisecular de la Iglesia orante. Ofrece su peculiar spiritalis ruminatio de la Escritura»: F. M. Arocena, La celebración de la palabra. Teología y pastoral, Barcelona, 2005, 35.

11 «Es una interpretación homilética del milagro, compuesta en estilo sencillo, sin recurrir a simbolismos ni alegorías»: J. SANCHO, o. $c$., 100.

${ }^{12} \mathrm{La}$ unidad entre los Testamentos se comprende también en la gestualidad. En el Codex emilianensis IV se pregunta Cur dicitur Ispanos iungere palmas ambas cum nobis lectio Euangelii refertur, a lo que se responde: manus iungit Legi ueteri nouam iungit: M. FÉROTIN, Le liber mozarabicus sacramentorum et les manuscrits mozarabes, Paris, 1912, 904.

Hispania Sacra, LIX

120, julio-diciembre 2007, 459-467, ISSN: 0018-215-X 
quam livor bellaverat serpentinus

novello partu verbo caelesti

populos exuteret saeculi subsequentis;

alius deinde piscis est,

qui priscae legis debitum

in similitudinem peccatorum

captus a Petro staterem evomuit

quem Dominus et Salvator noster eundem Petrum

evangelica voce censurae publicae imperat exolvendum

et pax caelestibus terris reddetur

Los dos peces de la perícopa evangélica significan los dos Testamentos ${ }^{13}$. El primero se asocia a la narración del libro de Tobías: la expulsión del demonio de Raquel $($ Tob 8, 2) y la curación de la ceguera de Tobit (Tob 3,17). No es difícil ver en estos dos momentos un paralelismo con el contenido de la Post Nomina. Además de esto, las citas de Tobías se presentan como parte de la ignorancia de los primeros siglos, después de la acción de la serpiente -justificada por la cita de Tob 8,2-, pero que por medio del parto del Verbo celeste quedó eliminada, pues Él devuelve la luz a los pueblos de los tiempos de los siglos futuros, esto es, a la Iglesia ${ }^{14}$. El primer pez, de este modo, nos transmite las acciones del demonio y de Cristo:

\section{Acción diabólica $\rightarrow$ Ignorancia \\ Acción de Cristo $\rightarrow$ Iluminación}

También esta comparación guarda relación con la interpretación adecuada de la Escritura: el demonio introduce la incapacidad de interpretar correctamente la Escritura, mientras que el Verbo encarnado ilumina (bautismo) al Nuevo Pueblo para que comprenda la Palabra escrita ${ }^{15}$.

${ }^{13}$ La asociación del número dos con la dualidad de los Testamentos la encontramos en varios autores medievales.

14 «La Iglesia no es, como lo era la Ley, un pedagogo del que precisa la adolescencia, pero del que podría prescindir la edad madura. «La educación divina», que ella tiene la misión de proporcionarnos, tiene la misma duración que el tiempo mismo»: H. DE LuBAC, Meditación sobre la Iglesia, Madrid, $21988,164$.

15 Una visión eucarística de la comprensión de las Escrituras: Sicque per totam Ecclesiam credentibus creditus, credituris credendus appareat, ut nullis per caecitatem erroris aut ignorantibus lateat, aut dubitantibus evanescat. Nec videndi stultitiam, nec credendi moram commotus accuset, sed ita sanctarum Scripturarum secreta mysteria indita nobis sapientiae facultate imprimens, profectum doc- 
El segundo pez hace alusión al pasaje de Mt 17, 25-27, en donde Cristo manda a Pedro a pagar el impuesto para no escandalizar, aunque era injusto. La solución de una posible disputa es comprendida en la Ad Pacem como una manera de introducir en la tierra la paz celeste, englobando de esta manera toda la reflexión de los sentidos espirituales de Tobías y Mateo dentro del tema de la paz, propio de esta oración.

En la illatio observamos una «alegoría mucho más discreta» ${ }^{16}$ :

Dignum et iustum est

virtutem Domini Iesu Christi mirabiliter enarrare,

qui populum defessum in suo saeculo

verbo dignatus est liberare.

Interrogat suos discipulos:

«Quos panes habetis?» 17

Ut seipsum manifestaret populis universis;

dicunt ei reverentes:

«Habemus pisciculos duos cum panibus quinque».

et pusillum est,

ut milia hominum exinde saturentur.

La función de la illatio es narrar el admirable poder de Cristo, haciendo uso de los versículos 38 y 42 de Mc 6, aunque sin descuidar los paralelos en otros evangelios ${ }^{18}$. En este momento la narración hace uso literal de la Sagrada Escritura $^{19}$. Del mismo modo que cuando un autor quiere interpretar un texto en su sentido espiritual, primero cita -aunque sea el incipit- el texto que desea comentar, así al comienzo de la illatio, para dar paso al sentido espiritual.

Duobus piscibus duos testamenta divina

Deus voluit demostrari,

tor aperiat; et, in virtute caelestis panis benedicti Corporis sui fragmenta distribuat ut in vivificatione animarum divini verbi et cibi alimonia concurrente, nec instructionis nobis desit copia, nec salutis: MHM, I, In IV feria Paschae, Oratio Admonitionis.

16 J. SANCHO, o. c., 100.

${ }^{17}$ Hemos agregado comillas a los diálogos entre Cristo y sus discípulos.

${ }^{18} \mathrm{El}$ término milia parece inspirarse en Lc 9, 14.

19 Por medio de una citación directa e indirecta: Cfr. G. RAmis MiQuel, Los misterios de pasión, como objeto de la anámnesis en los textos de la misa del rito hispánico. Estudio bíblico-teológico, Roma, 1978, 31-34.

Hispania Sacra, LIX

120, julio-diciembre 2007, 459-467, ISSN: 0018-215-X 
et in panibus quinque

Moysi libris legem pandit nobis,

Reafirmando el simbolismo de los dos peces como los dos Testamentos, la illatio adjudica al número de los panes los cinco libros del Pentateuco. Esta adjudicación está subyugada al número cinco, pues la idea teológica que está detrás de la eucología es la unidad «inter-testamentaria».

Discipuli intra se de panibus inopia cogitabant, et Deus populo suo copiosam cibariam praeparabat.

Fatus est Dominus:

Ieiunos eos nolo dimittere, se incipiant in via deficere, sustinere iacturam.

Centenos aut quinquegenos

per convivium discumbere fecit, et ieiunantem populum sua praesentia esurientemque refecit,

Christus benedicebat turbis circunstantibus, et panis crescebat in manibus;

sacri discipuli baiulantes

per circuitum frangebant panem, et eum suis occulis centuplicari videbant;

quantum escam esuriens populus comedebat, tantum cibus in edentium ore crescebat.

A estos simbolismos se une el doble nivel salvífico de la narración evangélica: mientras los apóstoles se dejaban absorber por la escasez y trataban de razonar una salida terrenal, Dios se adelantaba preparando el abundante alimento. Lo que sigue es una «ampliación» del texto que a veces observamos en la eucología, que «completa» lo que falta a la narración evangélica en sus detalles.

Postquam comederunt panem angelorum, colligerunt duodecim cophinos fragmentorum.

«Colligite ne pereant», ait Dominus Iesus Christus 
discipulis suis,

qui etiam propter nos descendit de caelis,

panis vivus,

et est iudex et medicus vulnerorum nostrorum,

qui Dei Patris possideret regnum;

cui merito.

Aunque se sigue narrando y «completando» la escena evangélica, se introducen comprensiones tipológicas, es decir, se asocia lo sucedido con el milagro del maná por medio de la expresión 'pan que desciende del cielo' (Cfr. Ex 16, 4; Neh 9, 15; Jn 6, 31ss) y 'pan de ángeles', ambas expresiones equivalentes (Cfr. Sab 16, 20).

En el Post Sanctus la alegoría vuelve a ser evidente ${ }^{20}$ :

Vere sanctus,

vere benedictus

Dominus noster Iesus Christus,

qui esurientem multitudinem

in quinque panibus et duobus piscibus

cibum esse dignatus est,

non ut milia convenientiam,

quod factum mirifice

sacra evangeliorum testantur eloquia,

sed per librorum voluminibus quinque

per quibus insatiata populis pectora

Moyse canente replentur,

proinde apostolis congregatum

iussit faenum adspergere,

id ignorantiam plebis replendam

dominicis praeparare praeceptis.

20 «Vuelve a prevalecer el alegorismo en la interpretación que la PS $(20,6)$ hace del episodio evangélico. Las últimas palabras (id ignorantiam plebis replendam dominicis praeparare praeceptis) aluden al llamado «Sermón eucarístico» según $J n$ 6, y serían así también una correcta transición a la anámnesis de la Cena»: J. SANCHO, o. c., 101.

Hispania Sacra, LIX

120, julio-diciembre 2007, 459-467, ISSN: 0018-215-X 
La acción de Cristo no se debe a la conveniencia de la multitud que tenía hambre, sino a la plenificación de la figura del pan del cielo del libro del Éxodo, en donde el Post Sanctus aprecia el corazón hambriento de los tiempos mosaicos, inspirándose seguramente en Jn $6,50 \mathrm{~s}^{21}$. En cualquier caso, los acontecimientos del Antiguo Testamento preparaban el precepto dominical, esto es, la celebración eucarística ${ }^{22}$.

Los textos eucológicos que hemos analizado brevemente, sin duda se corresponden con las funciones propias de su género ${ }^{23}$, pero nos recuerdan sin dificultad los comentarios exegéticos de los Padres. En este sentido, estos textos son excepcionales, pues la liturgia reinterpreta la Escritura de forma más sintética, más orante que indagante. No obstante, el estilo de nuestros textos revela un aspecto importante de la oración cristiana: también reflexionando se ora; la lectio divina es oración en sentido estricto. Además de este dato, nos damos cuenta que la liturgia tiende a reinterpretar, uniendo, toda la historia salutis.

La ausencia de este tipo de formularios, ya sea en el Missale Mixtum o en el MHM, nos muestran que las predilecciones respecto a la forma y el contenido de la eucología se han ido uniformando con el paso del tiempo, empobreciendo a veces nuestro conocimiento de la variabilidad de los textos y haciendo que olvidemos los vínculos profundos entre la oración litúrgica y la exégesis bíblicopatrística. La liturgia, incluso limitándonos a los textos, nunca nos deja de sorprender.

21 «Este es el pan que baja del Cielo, para que si alguien come de él no muera. Yo soy el pan vivo que he bajado del Cielo. Si alguno come de este pan vivirá eternamente; y el pan que yo daré es mi carne para la vida del mundo».

${ }^{22}$ Son muchos los textos que expresan esta idea en el MHM. Por ejemplo: Praeceptorum tuorum, Domine, mysteria recolentes, quaesumus, oblationis huius libamina sanctificare digneris: MHM, I, In sexto Dominico de Adventu, Post Pridie.

${ }^{23}$ Esto es, las illationes tienen su estilo de illationes, con su protocolo, embolismo y escatocolo, etc. 